Title: An early case of a natural barrier to axonal degeneration

Author: Axel Petzold

Sir, axonal transection has been described as the driving force for neurodegeneration in multiple sclerosis [1]. The seminal histological observation made hasve since been confirmed independently with aid of many structural and functional techniques [2]. Importantly the process of neurodegeneration can be transmitted trans-synaptically in multiple sclerosis and stroke amongst others $[3 ; 4]$. The end result of this process is optic nerve atrophy. Every physician will have to acquire during medical training the bedside skills to recognise a "pale disc" as the potential harbinger of potentially lifve threatening pathology. This skill has remained with our profession ever since the discovery invention of the ophthalmoscope.

It is worth the while to recall a histological post-mortem observation made briefly in 1857 shortly after the ophthalmoscope was invented. The treating ophthalmologist had observed bilateral pale optic discs in a patient with a history of painless loss of vision in both eyes [5]. Alert to the potential relevance, a post-mortem examination of the retina, optic nerve and chiasm was performed. The detailed histological examination revealed complete loss of nerves at the optic disc. But more importantly, extending the analysis to the point of sharpest vision, the macular area, permitted to observeshowed that there was good preservation of the outer retinal layers, the photoreceptors.

The description of the cellular layers and their connection in the retina belongs probably to the most detailed ones of the human central nervous system (CNS). The retina is derived from the ventricles around the diencephalon. Similar to the development of the cerebral cortex, multipotent neuroblastic precursors differentiate and migrate to their laminar destination of the multilayered tissue. The outer retinal layer contains the photosensitive tissue. The two cell types have been named by their shape as rods and cones. There are almost 100 million rods in the retina compared to about 4.5 million cones most of the latter are located in a small area called the macular. The innermost part of the macular only contains photoreceptors which, in absence of superimposed layers, can best be reached by light and therefore represents the area of sharpest vision. Moving away from the foveolar to the optic disc the multilayer appearance of the retina becomes more apparent. The photoreceptors convert light to an electric signal and their inner processes pass through the external limitating membrane to form the outer nuclear layer. This is the first of three nuclear layers, Heinrich Mueller refers to in his manuscript. The axons and synapses of the photoreceptors end in a pedicle (cone) or spherule (rod) which are in synaptic contact with dendrites of the bipolar and horizontal cells in the outer plexiform layer. Next are the inner nuclear layer, the second nuclear layer which contains the cell bodies of the bipolar cells, the amacrine cells, the Müller cells and the interplexiform cells. Again axons project to synapse with the dendrites of the retinal ganglion cells in the inner plexiform layer which continues into the inner nuclear layer, containing the retinal ganglion cells. The axons of the retinal ganglion cells finally project to the optic disc where they coalesce and leave the eye through the lamina cribrosa, after which the axons become myelinated to form the optic nerve. Functionally the three cellular layers of the retina and intersecting two plexiform layers convert an analogue light signal to a fine tuned digital signal. Most of the axons from the optic nerve reach the dorsal lateral geniculate nucleus were they synapse. Most neurons of dorsal lateral geniculate nucleus project their axons through the optic tracts and radiations to the primary visual cortex. 
The relevant three paragraphs of the observation (available in full lengths as supplementary material) are shown in Figure 1. The contemporary translation reads as follows.

"6. Anatomical finding in a case with amaurosis and atrophy of the optic nerve. [...] A while ago I had the opportunity to investigate the fresh and well preserved eyes of a person who suffered from painless onset of blindness loss of vision since over a few months. The observations made, are, as I believe extraordinary in several aspects. It is that I found almost complete atrophy of the nerve fibre layer and of the ganglion cell layer in the retina, whilst the there were hardly any changes to the remaining layers." (Figure $1 \mathrm{~A}$ )

The investigator, Heinrich Müller had two post-mortem eyes for investigation. One was fixed, the other was not. The findings were confirmatory for both tissue storage conditions.

"The optic nerve was at in this as well the other eye very much atrophied all the way to the chiasm. Unfortunately I do not know how much further back." (Figure 1 B)

At the time two conclusions were drawn. First, that there might be a mechanism of secondary peripheral or central axonal atrophy in the retina. This has is now recognised as antero- and retrograde trans-synaptic axonal degeneration [3; 4] following axonal transection [1]. Second that there might be a degree of independence of the photoreceptors.

"First, it is interesting for the understanding of the homoeostatic relationship of the elements of the retina, that atrophy affected only the ganglion cells, retinal nerve fibres and optic nerve. This implies that the outer retinal layers (from the inner nuclear layer onwards) are in this respect to a certain degree independent. Furthermore, it would be remarkable if atrophy of the ganglion cells was secondary to atrophy of the optic nerve. Unfortunately this cannot be determined, because I did not have the brain available for further assessments, I was therefore unable to investigate if atrophy advanced centrally or peripherally, which permits for the possibility that atrophy might have originated in the ganglion cells." (Figure $1 \mathrm{C}$ )

It is the first of these two conclusions which remains relevant because it implies the presence of a natural barrier to trans-synaptic axonal degeneration. Consistent with this historical observation is that a theoretically possible inexorabley "domino effect" of trans-synaptic axonal degeneration, wiping out the entire CNS has never been reported. What has been demonstrated is that retrograde trans-synaptic axonal degeneration affects certain pathways such as the optic radiations, optic tract, dLGN, optic nerve, retinal nerve fibre layer and retinal ganglion cells $[3,4]$. A review of the recent literature shows that retrograde axonal degeneration of the visual pathways, independent tof aetiology, stops at level of the inner nuclear layer [6].

It might potentially be a great gain, for the management of neurodegeneration, to better understand the mechanisms by which trans-synaptic axonal degeneration is brought to a halt. This is presents also a new opportunity for It is however an unlucky coincidence that this natural barrier falls between two different medical specialitiesophthalmology and neurology to work together. There are a number of ophthalmological conditions affecting the outer retinal layers which permit to study anterograde neurodegeneration reaching the INL. Likewise there are many neurological conditions of the anterior and posterior pathways which permit to study retrograde (transsynaptic) neurodegeneration. Paradoxically, the strength of this natural barrier has been such that 
diseases and with them ophthalmology and neurology could developed independently on either side of it.

The historical timing of this observation coincides with the discovery of Wallerian degeneration in the frog (1850) and the first description of axonal transection in the central nervous system of patients with multiple sclerosis by Charcot (1868).

\section{Conflict of interest statement}

The authors report no conflict of interest.

\section{Funding}

This study was not funded.

\section{References}

[1] Trapp, B.; Peterson, J. and others (1998). Axonal transection in the lesions of multiple sclerosis., N Eng J Med $338:$ 278-285.

[2] Friese, M. A.; Schattling, B. and Fugger, L. (2014). Mechanisms of neurodegeneration and axonal dysfunction in multiple sclerosis., Nat Rev Neurol : 225-238.

[3] Jindahra, P.; Petrie, A. and Plant, G. T. (2009). Retrograde trans-synaptic retinal ganglion cell loss identified by optical coherence tomography., Brain 132 : 628-634.

[4] Gabilondo, I.; Martinez-Lapiscina, E. H.; Martinez-Heras, E.; Fraga-Pumar, E.; Llufriu, S.; Ortiz, S.; Bullich, S.; Sepulveda, M.; Falcon, C.; Berenguer, J.; Saiz, A.; Sanchez-Dalmau, B. and Villoslada, P. (2014). Trans-synaptic axonal degeneration in the visual pathway in multiple sclerosis., Ann Neurol 75 : 98-107.

[5] Müller, H. (1857). Anatomische Beiträge zur Ophthalmologie, Archiv für Ophthalmologie. 1-98.

[6] Balk, L. J. and Petzold, A. (2014). Current and future potential of retinal optical coherence tomography in multiple sclerosis with and without optic neuritis., Neurodegener Dis Manag 4 : 165-176. 